DRAFT VERSION NOVEMBER 1, 2018

Preprint typeset using LTEX style emulateapj v. 08/22/09

\title{
ALFVÉN WAVE REFLECTION AND TURBULENT HEATING IN THE SOLAR WIND FROM 1 SOLAR RADIUS TO 1 AU: AN ANALYTICAL TREATMENT
}

\author{
Benjamin D. G. Chandran ${ }^{1}$ \& Joseph V. Hollweg ${ }^{1}$ \\ Draft version November 1, 2018
}

\begin{abstract}
We study the propagation, reflection, and turbulent dissipation of Alfvén waves in coronal holes and the solar wind. We start with the Heinemann-Olbert equations, which describe non-compressive magnetohydrodynamic fluctuations in an inhomogeneous medium with a background flow parallel to the background magnetic field. Following the approach of Dmitruk et al., we model the nonlinear terms in these equations using a simple phenomenology for the cascade and dissipation of wave energy, and assume that there is much more energy in waves propagating away from the Sun than waves propagating towards the Sun. We then solve the equations analytically for waves with periods of hours and longer to obtain expressions for the wave amplitudes and turbulent heating rate as a function of heliocentric distance. We also develop a second approximate model that includes waves with periods of roughly one minute to one hour, which undergo less reflection than the longerperiod waves, and compare our models to observations. Our models generalize the phenomenological model of Dmitruk et al. by accounting for the solar wind velocity, so that the turbulent heating rate can be evaluated from the coronal base out past the Alfvén critical point - that is, throughout the region in which most of the heating and acceleration occurs. The simple analytical expressions that we obtain can be used to incorporate Alfvén-wave reflection and turbulent heating into fluid models of the solar wind.
\end{abstract}

Subject headings:

\section{INTRODUCTION}

Beginning with the discovery of large-amplitude Alfvén waves in the interplanetary medium four decades ago (Belcher, Davis, \& Smith 1969; Belcher \& Davis 1971), spacecraft measurements have demonstrated that turbulent fluctuations in the magnetic field and plasma velocity are ubiquitous in the solar wind (see, e.g., Tu \& Marsch 1995; Goldstein, Roberts, \& Matthaeus 1995; Bruno \& Carbone 2005). As in hydrodynamic turbulence, fluctuation energy in turbulent magnetized plasmas cascades from large scales to small scales. Upon reaching sufficiently small scales (typically, of order the proton gyroradius and smaller), the energy is dissipated, thereby heating the ambient plasma [see Schekochihin et al. (2009) for a recent and detailed discussion]. Turbulent heating of the solar wind is thus inevitable, and may play an important role in the origin and evolution of the solar wind (see, e.g., Coleman 1968; Barnes 1981; Hollweg 1983; Tu et al. 1984; Hollweg 1986; Tu 1987, 1988; Hollweg \& Johnson 1988; Velli et al. 1989, 1990; Grappin and Velli 1991; Velli 1991; Grappin et al. 1993; Marsch and Tu 1996; Matthaeus et al. 1999).

The bulk of the fluctuation energy in solar-wind turbulence is at length scales greatly exceeding the proton gyroradius, is non-compressive, and to a good approximation can be treated within the approximation of reduced magnetohydrodynamics (MHD) (Kadomtsev \& Pogutse 1974; Strauss 1976; Zank \& Matthaeus 1992; Schekochihin et al 2009). The cascade of energy to smaller scales in reduced MHD turbulence arises only from interactions between oppositely propagating Alfvén waves. It has thus been known for some time that the launching of outward-propagating Alfvén waves by the Sun is not sufficient to cause turbulent heating, unless some addi-

\footnotetext{
${ }^{1}$ Space Science Center and Department of Physics, University of New Hampshire, Durham, NH; benjamin.chandran@unh.edu, joe.hollweg@unh.edu
}

tional process generates Alfvén waves that propagate towards the Sun (in the solar-wind frame). Different mechanisms for producing Sunward-propagating waves have been considered, including the parametric instability (Galeev \& Oraevskii 1963; Viñas \& Goldstein 1991; Del Zanna, Velli, \& Londrillo 2001 ) and instabilities driven by velocity shear (Roberts et al. 1992; Breech et al., 2008). In this paper, we focus on a third mechanism: the non-WKB reflection of Alfvén waves.

When the non-uniformity of the background plasma is taken into account, purely outward propagating waves are no longer a solution to the reduced MHD equations. Instead, outward and inward waves are coupled through linear terms that are proportional to the gradients in the Alfvén speed (Velli 1993; Hollweg \& Isenberg 2007). This coupling gives rise to wave reflection. At sufficiently high frequencies, linear Alfvén waves propagating in a hypothetical, steady solar wind are insensitive to radial variations in the Alfvén speed and undergo little reflection. On the other hand, for wave periods exceeding $\sim 20$ minutes, the transmission coefficient for outward-propagating Alfvén waves launched from the Sun drops significantly below unity (Velli 1993). A number of authors (e.g., Velli et al. 1989; Matthaeus et al. 1999; Dmitruk et al. 2001; Dmitruk \& Matthaeus 2003; Chandran et al. 2009; Verdini et al. 2009) have discussed the propagation of Alfvénic fluctuations originating at the Sun, emphasizing the self-consistent generation of reflected waves and the resulting cascade and dissipation of wave energy. In particular, Dmitruk et al. (2002, hereafter "D02") developed a simple phenomenological model describing reflections and turbulence and obtained an analytical approximation for the turbulent heating rate valid close to the Sun in the limit of strong turbulent dissipation. Elements of their approach were incorporated into detailed solar wind models by Cranmer \& van Ballegooijen (2005, hereafter CvB05), Cranmer et al. (2007), and Verdini \& Velli (2007, hereafter VV07).

In view of the potential usefulness of D02's analytical ap- 
proximation, our purpose here is to extend their approximation out to the Alfvén critical point and beyond, i.e., throughout the region where the principal solar wind heating and acceleration occur, and to obtain analytical formulas for the radial profiles of the turbulent heating rate and wave amplitudes. In section 2 we focus on waves with periods of hours and longer. In section 3 , we extend our model to account for shorter-period waves.

\section{LOW-WAVE-FREQUENCY MODEL}

We begin with the usual equations of ideal MHD,

$$
\begin{gathered}
\frac{\partial \rho}{\partial t}+\nabla \cdot(\rho \mathbf{v})=0 \\
\rho\left(\frac{\partial}{\partial t}+\mathbf{v} \cdot \nabla\right) \mathbf{v}=-\nabla p_{\text {tot }}+\frac{\mathbf{B} \cdot \nabla \mathbf{B}}{4 \pi}-\rho \nabla \Phi
\end{gathered}
$$

and

$$
\frac{\partial \mathbf{B}}{\partial t}=\nabla \times(\mathbf{v} \times \mathbf{B})
$$

where $\rho, \mathbf{v}$, and $\mathbf{B}$ are the mass density, velocity, and magnetic field, $\Phi$ is the gravitational potential, $p_{\text {tot }}=p+B^{2} / 8 \pi$ is the total pressure, and $p$ is the plasma pressure. We set

$$
\mathbf{v}=\mathbf{U}+\delta \mathbf{v}
$$

and

$$
\mathbf{B}=\mathbf{B}_{0}+\delta \mathbf{B},
$$

neglect the Sun's rotation, and take the background flow velocity $\mathbf{U}$ to be aligned with the background magnetic field $\mathbf{B}_{0}$. We assume that $\delta \mathbf{v}$ and $\delta \mathbf{B}$ are perpendicular to $\mathbf{B}_{0}$ and noncompressive. We neglect density fluctuations ${ }^{2}$ and take $\rho, \mathbf{U}$, and $\mathbf{B}_{0}$ to be steady-state solutions of equations (1) through (3). The vector Alfvén velocity is given by

$$
\mathbf{v}_{\mathrm{A}}=\frac{\mathbf{B}_{0}}{\sqrt{4 \pi \rho}},
$$

and the Elsässer variables are defined as

$$
\mathbf{z}^{ \pm}=\delta \mathbf{v} \mp \delta \mathbf{b},
$$

where $\delta \mathbf{b}=\delta \mathbf{B} / \sqrt{4 \pi \rho}$. Re-writing equations (2) and (3) in terms of $\mathbf{z}^{ \pm}$, we obtain (Velli 1993; VV07)

$$
\begin{gathered}
\frac{\partial \mathbf{z}^{ \pm}}{\partial t}+\left(\mathbf{U} \pm \mathbf{v}_{\mathrm{A}}\right) \cdot \nabla \mathbf{z}^{ \pm}+\mathbf{z}^{\mp} \cdot \nabla\left(\mathbf{U} \mp \mathbf{v}_{A}\right) \\
+\frac{1}{2}\left(\mathbf{z}^{-}-\mathbf{z}^{+}\right)\left(\nabla \cdot \mathbf{v}_{\mathrm{A}} \mp \frac{1}{2} \nabla \cdot \mathbf{U}\right)=-\left(\mathbf{z}^{\mp} \cdot \nabla \mathbf{z}^{ \pm}+\frac{\nabla p_{\text {tot }}}{\rho}\right) .
\end{gathered}
$$

The $\nabla p_{\text {tot }}$ term cancels the compressible part of the remaining terms in equation (8) to maintain the incompressibility condition $\nabla \cdot \mathbf{z}^{ \pm}=0$.

To proceed further, we assume that the background magnetic field $\mathbf{B}_{0}$ possesses a field line that is purely radial. This field line can be at any heliographic latitude. We then consider a cylindrical coordinate system $(R, \phi, z)$ whose $z$ axis is aligned with this radial field line, and we restrict our analysis to the region close to the $z$ axis, in which we assume that

\footnotetext{
2 To make this assumption self-consistent, we must also neglect spatial
} variations in the background density in the directions perpendicular to $\mathbf{B}_{0}$.
$v_{A \phi}=U_{\phi}=0$ and $\partial U / \partial \phi=\partial v_{\mathrm{A}} / \partial \phi=0$. Near the $z$ axis, the components of $\mathbf{U}$ and $\mathbf{v}_{\mathrm{A}}$ can be expanded in powers of $R$,

$$
\begin{aligned}
U_{R} & =a_{1}(z) R+\ldots, \\
v_{\mathrm{A}, R} & =a_{2}(z) R+\ldots
\end{aligned}
$$

Keeping just the first terms in these Taylor series, we find that $\partial U_{R} / \partial R=U_{R} / R, \partial v_{\mathrm{A}, R} / \partial R=v_{\mathrm{A}, R} / R$, and

$$
\mathbf{z}^{\mp} \cdot \nabla\left(\mathbf{U} \mp \mathbf{v}_{\mathrm{A}}\right)=\frac{\mathbf{z}^{\mp}\left(U_{R} \mp v_{\mathrm{A}, R}\right)}{R} .
$$

We define

$$
\frac{\partial}{\partial s}=\left(\mathbf{B}_{0} / B_{0}\right) \cdot \nabla
$$

so that $s$ is distance along a background magnetic field line. Since $\nabla R=\hat{R}$,

$$
\frac{U_{R} \mp v_{\mathrm{A}, R}}{R}=\frac{\left(U \mp v_{\mathrm{A}}\right)}{R} \frac{\partial R(s)}{\partial s},
$$

where $R(s)$ is to be interpreted as the distance from the $z$ axis to a given background magnetic field line.

We define vector versions of the Heinemann \& Olbert (1980) variables $\mathbf{f}$ and $\mathbf{g}$ as follows:

$$
\begin{aligned}
& \mathbf{z}^{+}=\frac{\mathbf{g} \eta^{1 / 4}}{1+\eta^{1 / 2}} \\
& \mathbf{z}^{-}=\frac{\mathbf{f} \eta^{1 / 4}}{1-\eta^{1 / 2}}
\end{aligned}
$$

where

$$
\eta=\rho / \rho_{a},
$$

and $\rho_{a}$ is the value of $\rho$ at the Alfvén critical point (on the $z$ axis). Mass conservation requires that $\partial\left(\rho U / B_{0}\right) / \partial s=0$, and thus $\eta^{1 / 2}=v_{\mathrm{A}} / U$. With the use of equations (11) and (13), equation (8) can be re-written as (VV07) ${ }^{3}$

$$
\begin{aligned}
& \frac{\partial \mathbf{g}}{\partial t}+(U\left.+v_{\mathrm{A}}\right) \frac{\partial \mathbf{g}}{\partial s}+\left(U+v_{\mathrm{A}}\right) \mathbf{f} \frac{\partial L}{\partial s} \\
&=-\left(\frac{1+\eta^{1 / 2}}{\eta^{1 / 4}}\right)\left(\mathbf{z}^{-} \cdot \nabla \mathbf{z}^{+}+\frac{\nabla p_{\mathrm{tot}}}{\rho}\right) \\
& \frac{\partial \mathbf{f}}{\partial t}+\left(U-v_{\mathrm{A}}\right) \frac{\partial \mathbf{f}}{\partial s}+\left(U-v_{\mathrm{A}}\right) \mathbf{g} \frac{\partial L}{\partial s} \\
&=\left(\frac{\eta^{1 / 2}-1}{\eta^{1 / 4}}\right)\left(\mathbf{z}^{+} \cdot \nabla \mathbf{z}^{-}+\frac{\nabla p_{\text {tot }}}{\rho}\right),
\end{aligned}
$$

where $L=\ln \left[R(s) \eta^{1 / 4} / R_{\text {ref }}\right]$, and $R_{\text {ref }}$ is an arbitrary constant. Close to the $z$ axis, $\partial\left(B_{0} R^{2}\right) / \partial s=0$, and $R_{\text {ref }}$ can be chosen so that

$$
L=-\frac{1}{2} \ln \left(\frac{v_{\mathrm{A}}}{v_{\mathrm{A} a}}\right),
$$

where $v_{\mathrm{A} a}$ is the Alfvén speed at the Alfvén critical point on the $z$ axis.

Dmitruk et al. (2002) numerically solved equation (8) in the limit $U \rightarrow 0$. They found that the numerical results could

\footnotetext{
${ }^{3}$ Scalar versions of the linear terms in equations 17 and 18 (for just the $\phi$ components of $\mathbf{g}$ and $\mathbf{f}$ ) were obtained by Heinemann \& Olbert (1980), who took the fluctuations to be axisymmetric with $\mathbf{z}^{ \pm} \propto \hat{\phi}$.
} 
to a large extent be understood in terms of a phenomenological model in which $\mathbf{z}^{\mp} \cdot \nabla \mathbf{z}^{ \pm}$is replaced by $\left|z^{\mp}\right| \mathbf{z}^{ \pm} / 2 \lambda(s)$. We employ the same phenomenological model [see Chandran et al (2009) for a more detailed discussion], replacing the nonlinear terms $\mathbf{z}^{\mp} \cdot \nabla \mathbf{z}^{ \pm}+\rho^{-1} \nabla p_{\text {tot }}$ in equations (17) and (18) with $\left|z^{\mp}\right| \mathbf{z}^{ \pm} / 2 \lambda(s)$. Equations (17) and (18) then become

$$
\frac{\partial \mathbf{g}}{\partial t}+\left(U+v_{\mathrm{A}}\right) \frac{\partial \mathbf{g}}{\partial s}+\left(U+v_{\mathrm{A}}\right) \mathbf{f} \frac{\partial L}{\partial s}=-\frac{\eta^{1 / 4}}{2 \lambda}\left|\frac{f}{1-\eta^{1 / 2}}\right| \mathbf{g}
$$

and

$$
\frac{\partial \mathbf{f}}{\partial t}+\left(U-v_{\mathrm{A}}\right) \frac{\partial \mathbf{f}}{\partial s}+\left(U-v_{\mathrm{A}}\right) \mathbf{g} \frac{\partial L}{\partial s}=-\frac{\eta^{1 / 4}|g| \mathbf{f}}{2 \lambda\left(1+\eta^{1 / 2}\right)} .
$$

Observations (e.g. Belcher and Davis 1971) show that most of the Alfvénic power in the solar wind is at long periods, of the order of hours, as seen in the spacecraft frame. In this section, we thus restrict our attention to waves with periods of hours and longer in the frame of the Sun. We further assume that the amplitude of the outward propagating waves is sufficiently large, and $\lambda$ is sufficiently small, that the time scale for the cascade and dissipation of $z^{-}$fluctuations, $\lambda / z^{+}$, is shorter than the wave period. In this case, we can neglect the first and second terms on the left-hand side of equation (21) and solve equation (21) to obtain

$$
\mathbf{f}=\frac{2 \lambda(\eta-1) U}{\eta^{1 / 4}} \frac{\partial L}{\partial s} \hat{g},
$$

where $\hat{g}=\mathbf{g} / g$. We assume that the rapid cascading of $z^{-}$ energy leads to the inequality

$$
z^{-} \ll z^{+}
$$

so that we can neglect the third term on the left-hand side of equation (20). Taking the dot product of equation (20) with $\mathbf{g}$, and then taking the time average of the resulting equation, we find that

$$
\frac{\partial}{\partial s}\left\langle g^{2}\right\rangle=-2\left\langle g^{2}\right\rangle\left|\frac{\partial L}{\partial s}\right|,
$$

where $\langle\ldots\rangle$ denotes a time average. Since we restrict our analysis to the vicinity of our $z$ axis, $s \simeq r$, where $r$ is the radial coordinate in spherical coordinates centered on the Sun. We take $L$ to be a decreasing function of $r$ near the Sun, to have a minimum at $r=r_{m}$ (corresponding to the maximum of $v_{\mathrm{A}}$ ), and to increase with $r$ at $r>r_{m}$. We take $r_{m}$ to be less than the radius $r_{a}$ of the Alfvén critical point. Integrating equation (24), we find that

$$
\left\langle g^{2}\right\rangle^{1 / 2}=g_{a}\left(\frac{v_{\mathrm{A}}}{v_{\mathrm{A} a}}\right)^{1 / 2}
$$

for $r>r_{m}$, where $g_{a}$ is the rms value of $g$ at $r=r_{a}$. For $r<r_{m}$, we find that

$$
\left\langle g^{2}\right\rangle^{1 / 2}=g_{a}\left(\frac{v_{\mathrm{A} m}^{2}}{v_{\mathrm{A} a} v_{\mathrm{A}}}\right)^{1 / 2},
$$

where $v_{\mathrm{A} m}$ is the Alfvén speed at $r=r_{m}$.

The energy density of the Alfvénic fluctuations is $\rho\left[\left(z^{+}\right)^{2}+\right.$ $\left.\left(z^{-}\right)^{2}\right] / 4$. We assume that the energy that is drained from $z^{+}$ and $z^{-}$fluctuations by the nonlinear "damping" terms in our model is converted to thermal energy. The turbulent heating rate, for $z^{-} \ll z^{+}$, is thus

$$
Q=\frac{\rho\left|z^{-}\right|\left(z^{+}\right)^{2}}{4 \lambda} .
$$

With the use of equations (22), (25), and (26), we find that the average heating rate is

$$
Q=\frac{\rho \eta^{1 / 2} g_{a}^{2} U}{4\left(1+\eta^{1 / 2}\right) v_{\mathrm{A} a}}\left|\frac{d v_{\mathrm{A}}}{d r}\right|
$$

for $r>r_{m}$, and

$$
Q=\frac{\rho \eta^{1 / 2} g_{a}^{2} U}{4\left(1+\eta^{1 / 2}\right)}\left(\frac{v_{\mathrm{A} m}^{2}}{v_{\mathrm{A} a} v_{\mathrm{A}}^{2}}\right) \frac{d v_{\mathrm{A}}}{d r}
$$

for $r<r_{m}$.

To check the validity of some of our approximations and compare to observations, we consider a model solar wind in which the proton number density is given by equation (4) of Feldman et al. (1997), which describes coronal holes out to several solar radii, plus an additional $r^{-2}$ component:

$$
n(r)=\left(\frac{3.23 \times 10^{8}}{x^{15.6}}+\frac{2.51 \times 10^{6}}{x^{3.76}}+\frac{1.85 \times 10^{5}}{x^{2}}\right) \mathrm{cm}^{-3},
$$

where $x=r / R_{\odot}$ and $R_{\odot}=1$ solar radius. This leads to $n=$ $4 \mathrm{~cm}^{-3}$ at $1 \mathrm{AU}$. We take the magnetic field strength to be (Hollweg \& Isenberg 2002)

$$
B_{0}=\left[\frac{1.5\left(f_{\max }-1\right)}{x^{6}}+\frac{1.5}{x^{2}}\right] \text { Gauss, }
$$

where $f_{\max }$ is the usual super-radial expansion factor, which we set equal to 5 . The solar wind speed $U$ is given by flux conservation:

$$
U=9.25 \times 10^{12} \frac{\tilde{B}}{\tilde{n}} \mathrm{~cm} \mathrm{~s}^{-1},
$$

where $\tilde{B}$ is $B_{0}$ in Gauss and $\tilde{n}$ is $n$ in units of $\mathrm{cm}^{-3}$. Equation (32) implies a flow speed of $750 \mathrm{~km} \mathrm{~s}^{-1}$ and a proton flux of $3 \times 10^{8} \mathrm{~cm}^{-2} \mathrm{~s}^{-1}$ at $1 \mathrm{AU}$. The Alfvén critical point in this model is at $r_{a}=11.1 R_{\odot}$, and the maximum of $v_{\mathrm{A}}$ is at $r_{m}=1.60 R_{\odot}$. The solar wind speed and Alfvén speed are plotted in Figure 1 .

We set

$$
\lambda=\lambda_{0}\left[\frac{B\left(R_{\odot}\right)}{B}\right]^{1 / 2},
$$

where $B\left(R_{\odot}\right)=7.5 \mathrm{G}$ from equation (31), and $\lambda_{0}$ is a constant. Previous studies suggest a range of possible values for $\lambda_{0}$. One choice is to set $\lambda_{0}$ equal to the mean spacing between photospheric flux tubes. Following Spruit (1981), we take this mean spacing to be $R_{p} \sqrt{\pi B_{p} / B_{\text {avg }}}$, where $R_{p}=200 \mathrm{~km}$ and $B_{p}=1500 \mathrm{G}$ are the typical radius and magnetic field strength of a photospheric flux tube, and $B_{\text {avg }}$ is the average magnetic field at the coronal base above the flux-tube merging height. If we set $B_{\text {avg }}$ equal to be the value of $B_{0}$ in equation (31) as $x \rightarrow 1$ (which is $7.5 \mathrm{G}$ ), then $\lambda_{0}=5000 \mathrm{~km}$. On the other hand, D02 used $\lambda_{0}=3 \times 10^{4} \mathrm{~km}$ (comparable to the average size of supergranules), $\mathrm{CvB} 05$ considered values of $\lambda_{0}$ in the range $1-30 \times 10^{3} \mathrm{~km}$, and the quantity corresponding to our $\lambda_{0}$ in VV07 was $1.7 \times 10^{4} \mathrm{~km}$. We will consider several values of $\lambda_{0}$ in the range spanned by the above values.

To check whether we are justified in neglecting the terms that we have dropped in equations (20) and (21), it would be desirable to compare our approximate solutions to exact, time-dependent solutions of equations (20) and (21). However, such time-dependent solutions are beyond the scope 


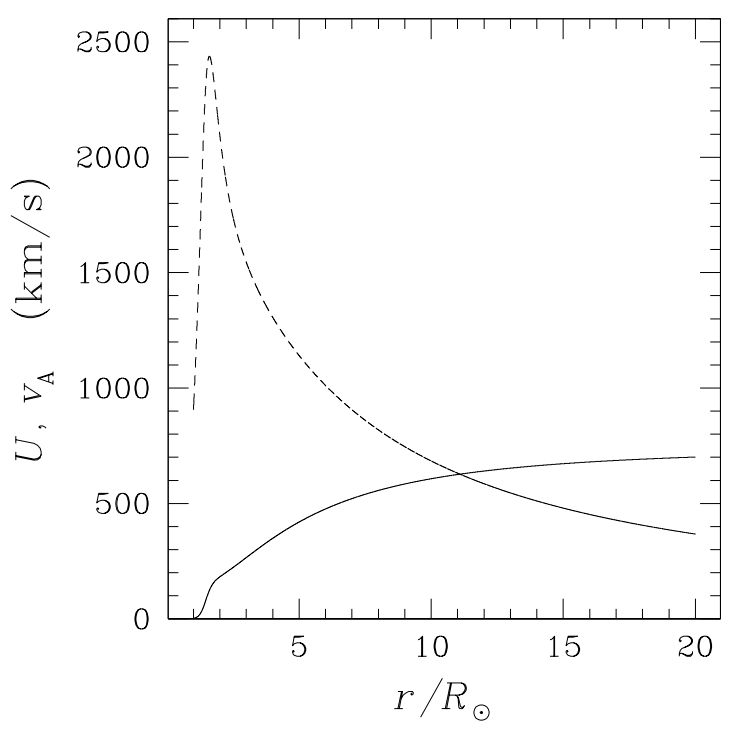

FIG. 1.- The solar wind velocity (solid line) and Alfvén speed (dashed line) from Equations 30] through [32 with $f_{\max }=5$.

of this study. Instead, we numerically solve the timeindependent versions of equations (20) and (21), taking $f=0$ at the Alfvén critical point, setting $\lambda_{0}=5000 \mathrm{~km}$ and $g_{a}=$ $8 \times 10^{7} \mathrm{~cm} / \mathrm{s}$, and assuming that the directions of $\mathbf{f}$ and $\mathbf{g}$ are the same and do not change with $s$. This steady-state numerical solution is still a useful reference point since we are considering only long-period oscillations in this section. In Figure 2, we plot the numerical solutions for $|f|$ and $g$, as well as our approximate analytical solutions from equations (22), (25), and (26). For $r>2 R_{\odot}$, our approximate solutions are close to the numerical solutions. Closer to the Sun, our analytical expressions over-estimate the values of $|f|$ and $g$. In particular, our approximate value of $g$ is $23 \%$ larger than the numerical solution at $r=R_{\odot}$. If $\lambda_{0}$ is increased to $10^{4} \mathrm{~km}$ $\left(1.5 \times 10^{4} \mathrm{~km}\right)$, the approximate solution is $43 \%(57 \%)$ larger than the numerical solution at $r=R_{\odot}$, although the approximate solutions remain reasonably accurate at $r>2 R_{\odot}$. Increasing $\lambda_{0}$ reduces the nonlinear terms in equations $(20)$ and (21) and degrades the accuracy of our approximations.

The loss of accuracy in our analytical approximations as $\lambda_{0}$ increases is further illustrated in Figure 3. which is analogous to Figure 5 of D02. The dashed line in this figure shows $Q / \rho$ from equations (28) and (29). The three solid lines plot the total heating rate $Q_{\text {total }}=\rho\left[\left(z^{+}\right)^{2}\left|z^{-}\right|+\left(z^{-}\right)^{2}\left|z^{+}\right|\right] / 4 \lambda$ in the numerical solutions of the time-independent versions of equations (20) and (21) for $g_{\mathrm{a}}=8.0 \times 10^{7} \mathrm{~cm} / \mathrm{s}$ and for three different values of $\lambda_{0}$. Going from the top of the figure to the bottom, these values are $2500 \mathrm{~km}, 5000 \mathrm{~km}$, and $10^{4} \mathrm{~km}$. At $r \geq 2 R_{\odot}$, equation (28) overestimates the numerical heating rate by $\lesssim 50 \%$ for the chosen values of $\lambda_{0}$. At $r<1.6 R_{\odot}$, equation (29) overestimates the numerical heating rate by almost an order of magnitude in the worst case $\left(\lambda_{0}=10^{4} \mathrm{~km}\right)$.

In Figure 4, we use our time-independent solutions of equations (20) and (21) with $g_{\mathrm{a}}=8.0 \times 10^{7} \mathrm{~cm} / \mathrm{s}$ and $\lambda_{0}=5000 \mathrm{~km}$ to plot each term in equation $(21)$, divided by $\left(1-\eta^{1 / 2}\right)$ to make the plot easier to read. Although we dropped $\partial f / \partial t$ to obtain these time-independent solutions, we estimate the

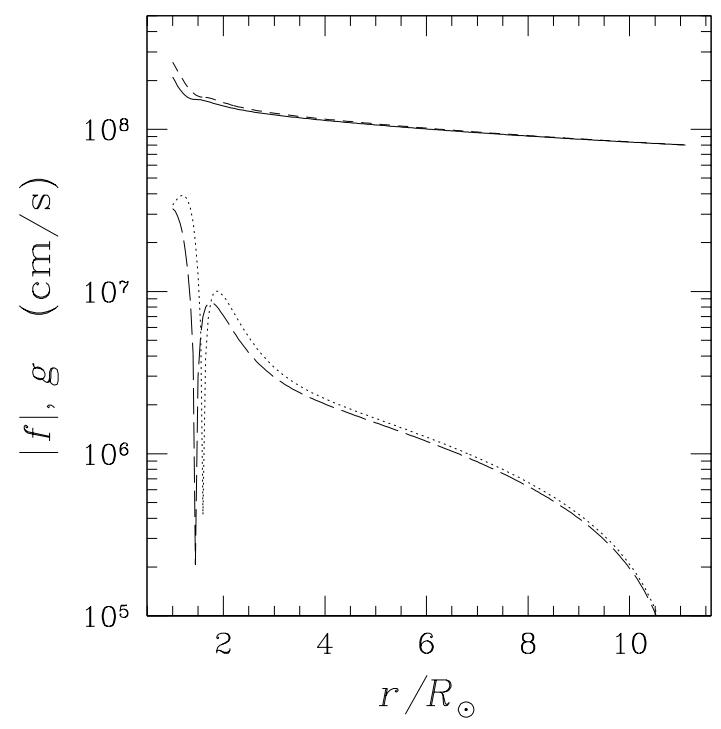

FIG. 2.- A comparison between our approximate solutions for $\mid f\lfloor$ (dotted line) and $g$ (short-dashed line) and numerical solutions to equations $(20)$ and (21) obtained in the limit of zero frequency. The solid line is the numerical solution for $g$ and the long-dashed line is the numerical solution for $|f|$. For these curves, we have taken $g_{\mathrm{a}}=8.0 \times 10^{7} \mathrm{~cm} / \mathrm{s}$ and $\lambda_{0}=5000 \mathrm{~km}$.

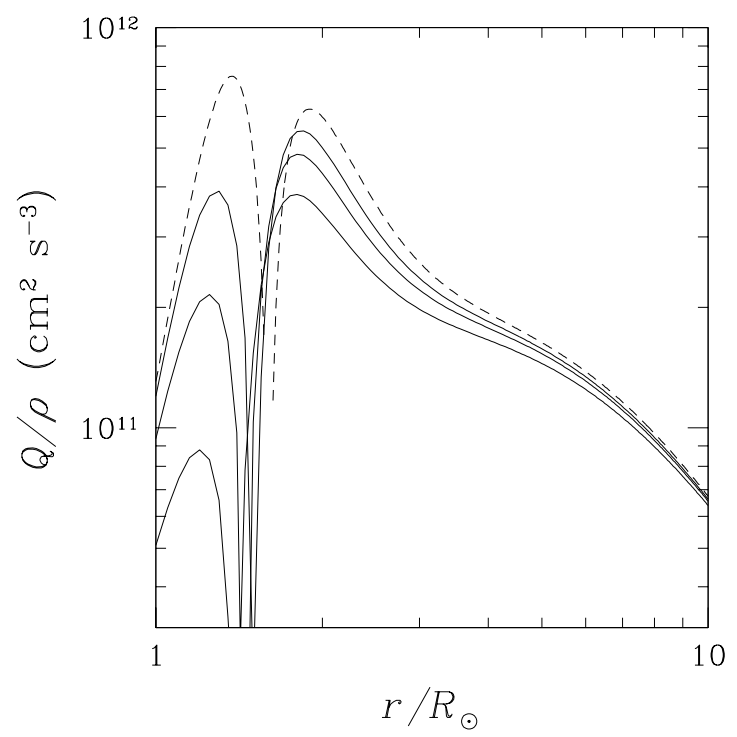

FIG. 3.- The dashed line is the analytical approximation of the heating rate per unit mass $Q / \rho$ from equations 28 and 29 , with $g_{\mathrm{a}}=8.0 \times 10^{7} \mathrm{~cm} / \mathrm{s}$. The solid lines are the total heating rate $Q_{\text {total }}=\rho\left[\left(z^{+}\right)^{2}\left|z^{-}\right|+\left(z^{-}\right)^{2}\left|z^{+}\right|\right] / 4 \lambda$ divided by $\rho$ in numerical solutions of the time-independent versions of equations 201 and 21, with $g_{\mathrm{a}}=8.0 \times 10^{7} \mathrm{~cm} / \mathrm{s}$ and with three different values of $\lambda_{0}: 2500 \mathrm{~km}, 5000 \mathrm{~km}$, and $10^{4} \mathrm{~km}$ (going from the top curve to the bottom curve). As $\lambda_{0}$ increases, our approximation becomes increasingly inaccurate.

approximate magnitude of the $\partial f / \partial t$ term by multiplying our numerical solution for $f$ by $2 \pi / P$, where $P$ is the wave period, which we take to be three hours. Our estimate of the $\partial f / \partial t$ term is smaller than the terms that we have kept inside the Alfvén critical point, but $\partial f / \partial t$ increases relative to the dom- 


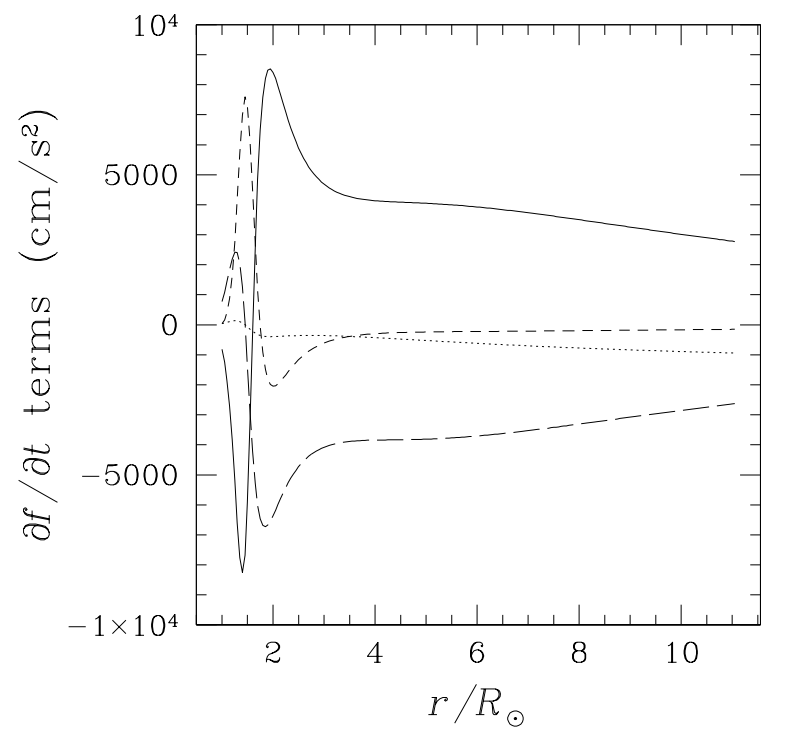

FIG. 4.- The different terms in equation 21 , divided by $\left(1-\eta^{1 / 2}\right)$ (see text). The solid line is the term proportional to $\partial L / \partial s$, the long-dashed line is the nonlinear term on the right-hand side of equation 21 , the dotted line is the $\partial f / \partial t$ term, and the short-dashed line is the $\partial f / \partial s$ term. As in figure 2 we have taken $g_{\mathrm{a}}=8.0 \times 10^{7} \mathrm{~cm} / \mathrm{s}$ and $\lambda_{0}=5000 \mathrm{~km}$.

inant terms as $r$ increases towards $r_{a}$. We have compared the different terms in equation (21) at larger $r$, and find that our neglect of the $\partial f / \partial t$ term becomes unjustified for $r \gtrsim 20 R_{\odot}$, because the nonlinear time scale becomes larger than the wave period (measured in the Sun's frame) due to the increase in $\lambda$ and the decrease in $z^{+}$as $r$ increases. Figure 4 shows that the $\partial f / \partial s$ term, which we have neglected in equation (21), is negligible except in a small region near $r=r_{m}$, where our solution for $f$ passes through zero. Although it is not plotted, we have also carried out the same comparison for the different terms in equation (20), using our time-independent numerical solution. We find that the $\partial L / \partial s$ term that we neglect in equation (20) is indeed smaller than the other terms out to well beyond $20 R_{\odot}$, because $f \ll g$.

In Figure 5, we plot the turbulent heating rate in equations (28) and (29) (thin solid line). Although the approximations leading to equations (28) and (29) break down at $r \gtrsim 20 R_{\odot}$, we plot equation $(28)$ out to beyond $100 R_{\odot}$ to illustrate how the analytical formulas extrapolate to larger radii. The heating rate in our model depends on a single free parameter, $g_{a}$, which we set equal to $8.0 \times 10^{7} \mathrm{~cm} / \mathrm{s}$ in order to roughly match two previous empirically constrained models of plasma heating in the fast solar wind (Allen et al. 1998; Cranmer et al. 2009). We note that for $z^{-} \ll z^{+}$, this value of $g_{a}$ corresponds to a fluctuating velocity of $200 \mathrm{~km} / \mathrm{s}$ at the Alfvén critical point. The dashed line in Figure 5 is the total (electron plus proton) heating rate $Q_{\mathrm{A} 98}$ in model SW2 of Allen et al. (1998). The thick solid line is the proton heating rate in the model of Cranmer et al. (2009) (their Eq. 14, which applies at $r>0.3 \mathrm{AU})$, multiplied by $4 / 3$ to convert the proton heating rate to an approximate total heating rate, denoted $Q_{\mathrm{C} 09}$. [The proton heating rate is typically $60-90 \%$ of the total heating rate in Cranmer et al's (2009) model].

Model SW2 of Allen et al. (1998) does not provide a tight constraint on the heating rate, since widely different heat-

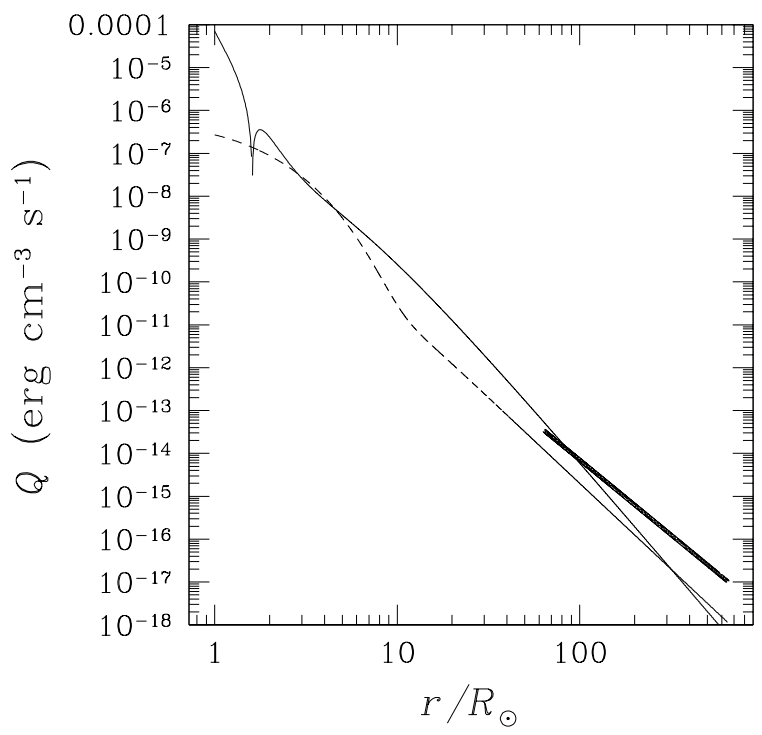

FIG. 5.- The thin solid line is the value of $Q$ in equations 28 and 29] for $g_{\mathrm{a}}=8.0 \times 10^{7} \mathrm{~cm} / \mathrm{s}$. The dashed line is the total (electron plus proton) heating rate in model SW2 of Allen et al. (1998). The thick solid line is the proton heating rate from Eq. (14) of Cranmer et al. (2009), multiplied by 4/3 to convert it to an approximate total heating rate (the proton heating rate is typically $60-90 \%$ of the total heating rate in their model).

ing functions and momentum-deposition profiles (their models SW2, SW3, and SW4) were shown to be consistent with in situ observations of the fast wind. We include $Q_{\mathrm{A} 98}$ in Figure 5 to show that our results for the heating rate at $2 R_{\odot}<$ $r<7 R_{\odot}$ are reasonably close to at least one empirically constrained model for a plausible value of $g_{a}$. On the other hand, our heating rate greatly exceeds $Q_{\mathrm{A} 98}$ at $r<1.5 R_{\odot}$. This disagreement may be due in part to the effects described in section 4 and also to the point illustrated in figure 3, that we substantially overestimate the heating rate close to the Sun when $\lambda_{0}>5000 \mathrm{~km}$. We emphasize that although Figure 5 represents a rough first attempt to compare our model to observational constraints on the heating rate, this comparison must be viewed with some caution, because the density profiles in the three models are different, and $Q$ depends upon $\rho$ in equations (28) and (29).

In Figure 6, we plot the rms amplitude of the fluctuating velocity in our low-wave-frequency model as a function of $r$ (dashed line), using equations (25) and (26) and the relation $\delta v_{\text {rms }} \simeq\left\langle\left(z^{+}\right)^{2}\right\rangle^{1 / 2} / 2$, which is valid when $z^{-} \ll z^{+}$. We also plot observed values and upper limits from remote UVCS observations of the corona (Esser et al. 1999; CvB05) and in situ measurements from Helios and Ulysses (Bavassano et al. 2000). (The solid line in this figure is from the extended model described in section 3.) For $g_{a}=8 \times 10^{7} \mathrm{~cm} / \mathrm{s}$, our low-wave-frequency model over-predicts the rms amplitude of velocity fluctuations at $1.5 R_{\odot}<r<2 R_{\odot}$. As noted above, the approximations leading to equations (25) and (26) break down at $r \gtrsim 20 R_{\odot}$. Nevertheless, we plot equation (25) out to larger $r$ to illustrate how the analytical formulas extrapolate to larger radii. This extrapolation shows that for $g_{a}=8 \times 10^{7} \mathrm{~cm} / \mathrm{s}$, equations (25) and (26) under-predict $\delta v_{\text {rms }}$ at $r>70 R_{\odot}$. 


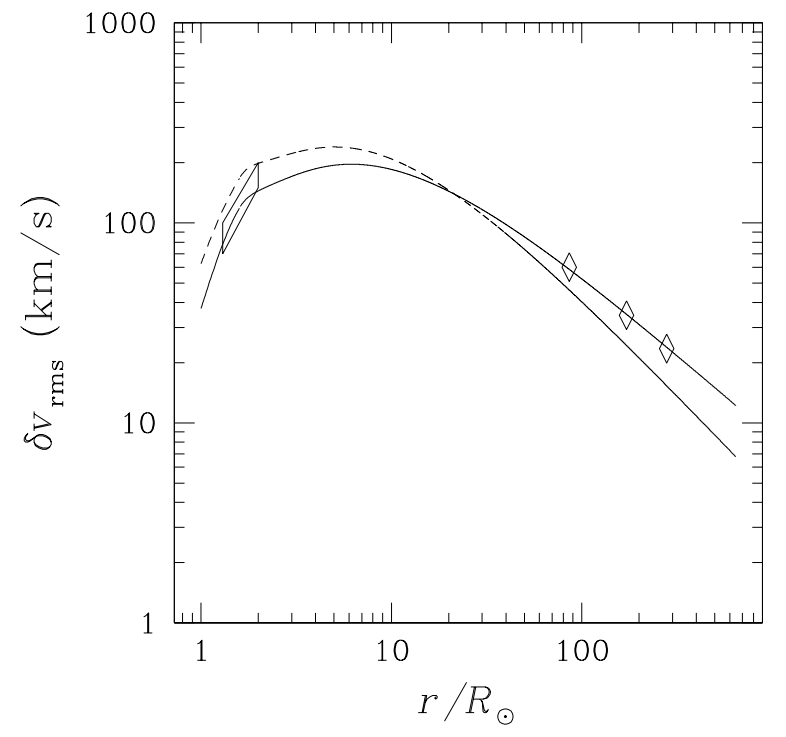

FIG. 6.- The dashed line is the rms value of the wave velocity in our low-wave-frequency model, which is approximately $z^{+} / 2$ when $z^{-} \ll z^{+}$, obtained from equations $(25)$ and 26 with $g_{a}=8 \times 10^{7} \mathrm{~cm} / \mathrm{s}$. The solid line is the rms value of the wave velocity in the extended model described in section 3 obtained from equations $(37)$ and 38 with $g_{a}=7.2 \times 10^{7} \mathrm{~cm} / \mathrm{s}$ and $\chi=0.65$. The open box is adapted from Figure 9 of $\mathrm{CvB} 05$, and represents an upper limit on the non-thermal line widths obtained by Esser et al. (1999) using off-limb UVCS measurements (Esser et al. 1999). The open diamonds are from in situ Helios and Ulysses measurements (Bavassano et al. 2000).

\section{EXTENDED MODEL ACCOUNTING FOR SHORTER-PERIOD WAVES}

In section 2, we took the waves launched by the Sun to have periods of hours and longer. On the other hand, CvB05 suggested that a significant fraction of the outward wave power near the Sun is in waves with periods of 1 minute to 1 hour. $^{4}$ In this section, we extend our model to account for such shorter-period waves in an approximate manner. The approximations we use are very simplistic, but are not limited to $r \lesssim 20 R_{\odot}$.

In the spirit of our simple, phenomenological modeling, we begin by replacing each $\mathbf{g}$ and $\mathbf{f}$ in equations (20) and (21) with the (time-independent) rms values $\left\langle g^{2}\right\rangle^{1 / 2}$ and $\left\langle f^{2}\right\rangle^{1 / 2}$, respectively. As shown by Velli (1993) and others, wave reflection becomes less efficient as the wave period decreases below $\sim 1$ hour, since higher-frequency waves with shorter parallel wavelengths are less affected by the radial gradient in the Alfvén speed. To account for this effect, we set

$$
\frac{\partial L}{\partial s} \rightarrow \chi \frac{\partial L}{\partial s}
$$

in equations (20) and 21, where $\chi$ is a dimensionless constant. In the low-wave-frequency limit of section $2, \chi \rightarrow 1$.

\footnotetext{
${ }^{4}$ We note, however, that VV07 considered two different frequency spectra for the $z^{+}$waves at the coronal base: a flat spectrum in which the energy is mostly in waves with periods $P$ of $\sim 10$ minutes (the small- $P$ cutoff in their spectrum), and a steep spectrum in which most of the energy is in the longest-period waves in their model, with $P \sim 10^{3}$ hours. With the steep spectrum, their model agreed with in situ measurements of the $z^{+}$and $z^{-}$ amplitudes, but with the flat spectrum their model over(under)-estimated the $z^{+}\left(z^{-}\right)$energy relative to the in situ measurements. The VV07 results thus favor a picture in which the Sun launches primarily long-period waves.
}

To model the mix of wave frequencies launched from the Sun, we leave $\chi$ as a free parameter between 0 and 1 . We take $\left\langle g^{2}\right\rangle^{1 / 2}$ and $\left\langle f^{2}\right\rangle^{1 / 2}$ to have scale lengths in the direction of $\mathbf{B}_{0}$ of order $r$. We continue to assume that $f \ll g$, and set $(\eta-1) \partial L / \partial s \rightarrow|(\eta-1) \partial L / \partial s|$ in equation (21) to maintain the positivity of $\left\langle f^{2}\right\rangle$, so that equation (21) becomes

$$
\left\langle f^{2}\right\rangle^{1 / 2}=\frac{2 \chi \lambda|\eta-1| U}{\eta^{1 / 4}}\left|\frac{\partial L}{\partial s}\right| .
$$

Dropping the third term on the left-hand side of equation 20. and using equation (35), we obtain

$$
\frac{\partial}{\partial s}\left\langle g^{2}\right\rangle=-2 \chi\left\langle g^{2}\right\rangle\left|\frac{\partial L}{\partial s}\right|,
$$

which we integrate to obtain

$$
\left\langle g^{2}\right\rangle^{1 / 2}=g_{a}\left(\frac{v_{\mathrm{A}}}{v_{\mathrm{A} a}}\right)^{\chi / 2}
$$

for $r>r_{m}$ and

$$
\left\langle g^{2}\right\rangle^{1 / 2}=g_{a}\left(\frac{v_{\mathrm{A} m}^{2}}{v_{\mathrm{A} a} v_{\mathrm{A}}}\right)^{\chi / 2}
$$

for $r<r_{m}$. From equation (27), we then find that

$$
Q=\frac{\chi \rho \eta^{1 / 2} g_{a}^{2} U}{4 v_{\mathrm{A}}\left(1+\eta^{1 / 2}\right)}\left(\frac{v_{\mathrm{A}}}{v_{\mathrm{A} a}}\right)^{\chi}\left|\frac{d v_{\mathrm{A}}}{d r}\right|
$$

for $r>r_{m}$, and

$$
Q=\frac{\chi \rho \eta^{1 / 2} g_{a}^{2} U}{4 v_{\mathrm{A}}\left(1+\eta^{1 / 2}\right)}\left(\frac{v_{\mathrm{A} m}^{2}}{v_{\mathrm{A} a} v_{\mathrm{A}}}\right)^{\chi} \frac{d v_{\mathrm{A}}}{d r}
$$

for $r<r_{m}$. The results of our low-frequency model in section 2 are recovered by setting $\chi=1$ in equations (35) through (40).

When $z^{-} \ll z^{+}$, the rms amplitude of the fluctuating velocity $\delta v_{\text {rms }}$ is approximately $\left\langle\left(z^{+}\right)^{2}\right\rangle^{1 / 2} / 2$, and there are two free parameters that determine $\delta v_{\mathrm{rms}}: g_{a}$ and $\chi$. We set $g_{a}=7.2 \times 10^{7} \mathrm{~cm} / \mathrm{s}$ and $\chi=0.65$ in order to match the observational constraints on the fluctuating velocity, shown in Figure 6. The solid line in Figure 6 is the resulting value of $\delta v_{\text {rms }}$ obtained from equations (37) and (38). In Figure 7 we plot the heating rate from equations (39) and (40) for these values of $g_{a}$ and $\chi$, along with the empirically constrained heating rates $Q_{\mathrm{A} 98}$ and $Q_{\mathrm{C} 09}$ from the models of Allen et al. (1998) and Cranmer et al. (2009) described in section 2 We plot the rms amplitudes of $z^{+}$and $z^{-}$resulting from equations (35), (37), and (38) in Figure 8, as well as in situ measurements from Helios and Ulysses (Bavassano et al. 2000). The rms amplitude of $z^{-}$that results from equation (35) depends upon both $\chi$ and $\lambda_{0}$. To match the in situ data, we set $\lambda_{0}=1.6 \times 10^{4} \mathrm{~km}$ in this figure, keeping $\chi=0.65$ as in Figures 6 and 7 As Figure 8 shows, the approximation $z^{-} \ll z^{+}$ fails at $r \gtrsim 1 \mathrm{AU}$.

Overall, the model matches the observations and previous models fairly well. However, as in our low-wave-frequency model, the heating rate in our extended model is much larger than $Q_{\mathrm{A} 98}$ at $r<1.5 R_{\odot}$. This discrepancy may arise in part for the reasons discussed in section 4 In addition, our extended model is likely similar to our low-frequency model in significantly overestimating $Q$ close to the Sun when $\lambda_{0} \geq 5000 \mathrm{~km}$ 


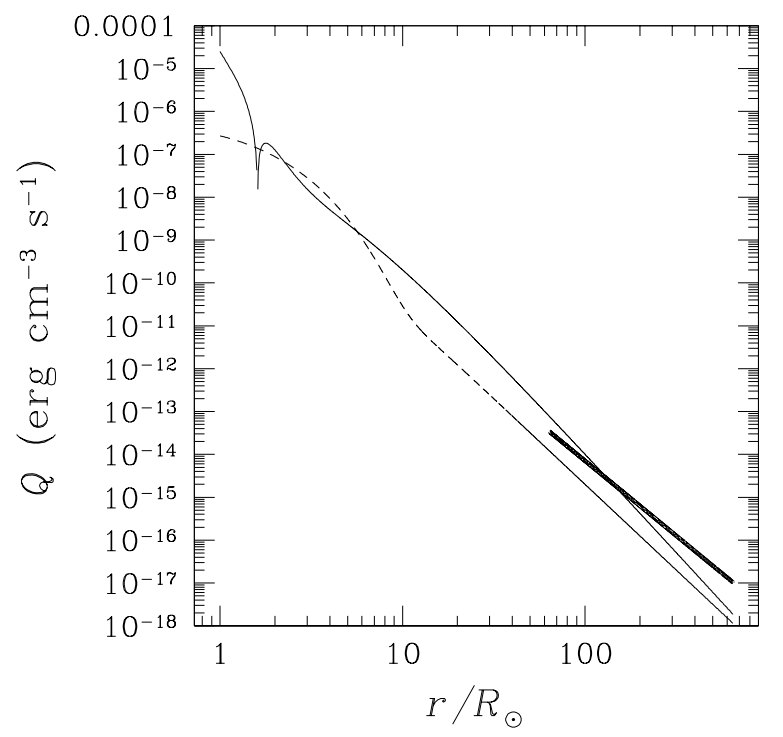

FIG. 7.- The solid line is the value of $Q$ in equations 39 and 40 for $g_{\mathrm{a}}=7.2 \times 10^{7} \mathrm{~cm} / \mathrm{s}$ and $\chi=0.65$. The dashed line is the total (electron plus proton) heating rate in model SW2 of Allen et al. (1998). The dotted line is the proton heating rate from Eq. (14) of Cranmer et al. (2009), which is typically $60-90 \%$ of the total heating rate in their model.

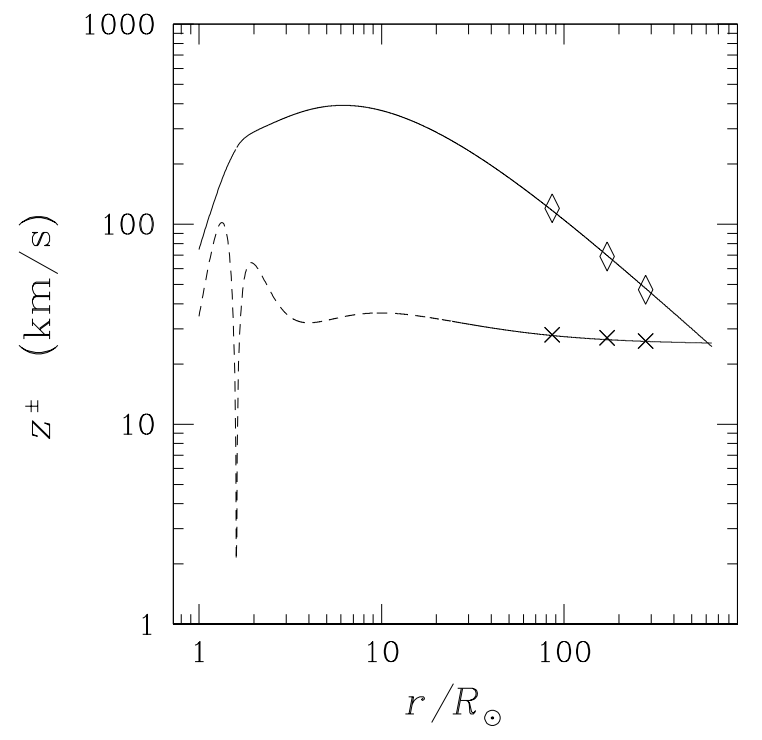

FIG. 8. - The solid line is the rms amplitude of $z^{+}$for $g_{a}=7.2 \times 10^{7} \mathrm{~cm} / \mathrm{s}$ and $\chi=0.65$ from equations 37 and 38 . The dashed line is the rms amplitude of $z^{-}$from equation 22 for $\lambda_{0}=1.6 \times 10^{4} \mathrm{~km}$ and the same value of $\chi$. The diamonds and crosses are the rms values of $z^{+}$and $z^{-}$, respectively, from Bavassano et al. (2000).

(Figure 3). A second difference between the heating rates plotted in Figure 7 is that $Q$ becomes smaller than $Q_{\mathrm{C} 09}$ at $r>1$ AU. This discrepancy may arise because of the increasing importance at large $r$ of sources of inward waves (such as velocity shear) that are not included in our model. We note, however, that some caution is warranted when comparing our heating rate to $Q_{\mathrm{A} 98}$ and $Q_{\mathrm{C} 09}$, because the density profiles in the three models are different, and $Q$ depends upon $\rho$ in equations (39) and (40).

Compared to our model for low-frequency waves in section 2, our extended model accounting for shorter-period waves does a notably better job of reproducing the observational constraints on the velocity fluctuations in the solar wind. This is not surprising, given that there is an additional free parameter in the extended model and the approximations underlying our low-wave-frequency model break down at $r \gtrsim 20 R_{\odot}$. There is, however, an additional reason for the closer agreement between the extended model and the data. As can be seen in Figure 6 the value of $\delta v_{\text {rms }}$ in our low-wavefrequency model drops off more rapidly with radius than does the data. When the efficiency of reflection is reduced, the rate at which the outward wave energy cascades and dissipates is reduced, and so $\left|(d / d r) \delta v_{\text {rms }}\right|$ decreases.

\section{THE ENERGY CASCADE TIME FOR $Z^{+}$WAVES}

If the Sun launches an outward-propagating $\left(z^{+}\right)$Alfvén wave packet with a perpendicular scale length $\lambda_{0}$ at the coronal base, with $\lambda_{0}$ greatly exceeding the dissipation scale, then this wave packet must propagate some distance into the corona before its energy cascades to small perpendicular scales and dissipates. We estimate this distance using approximations and results from our analytical models. As in equation (20), the rate at which $z^{-}$waves shear $z^{+}$waves is

$$
\gamma_{\mathrm{nl}}^{-}=\frac{\left|z^{-}\right|}{2 \lambda} \text {. }
$$

The quantity

$$
\Gamma=\int_{r_{b}}^{r} \frac{\left|z^{-}\right| d r_{1}}{2 \lambda\left(U+v_{\mathrm{A}}\right)}
$$

is the time integral of $\gamma_{\mathrm{nl}}^{-}[\mathbf{r}(t), t]$ evaluated at a position $\mathbf{r}(t)$ that moves outward with the $z^{+}$wave packet at speed $U+v_{\mathrm{A}}$, starting from the time the wave packet leaves the coronal base at $r=r_{b} \simeq 1 R_{\odot}$ until the time the wave packet reaches radius $r$. Roughly speaking, the $z^{+}$wave packet must propagate out to a distance at which $\Gamma \gtrsim 1$ before its energy cascades and dissipates. We evaluate the right-hand side of equation (42) in our low-frequency model using equation (22). To evaluate equation (42) using our extended-model results, we replace $z^{-}$ with $\left\langle\left(z^{-}\right)^{2}\right\rangle^{1 / 2}$ and use equation (35). The integral in equation (42) can then be evaluated analytically to yield

$$
\Gamma=\left\{\begin{array}{ll}
(\chi / 2) \ln \left(v_{\mathrm{A}} / v_{\mathrm{A} b}\right) & \text { if } r<r_{m} \\
(\chi / 2) \ln \left[v_{\mathrm{A} m}^{2} /\left(v_{\mathrm{A} b} v_{\mathrm{A}}\right)\right] & \text { if } r>r_{m}
\end{array},\right.
$$

where the result for our low-frequency model is obtained by setting $\chi=1$. The value of $\Gamma$ is plotted in Figure 9, assuming $\chi=1$ and using the profiles for $n, B_{0}$, and $U$ given in equations (30) through (32). The condition $\Gamma>1$ only holds for $r \gtrsim 7 R_{\odot}$, suggesting that the turbulent heating rate may be smaller than in our model within a few $R_{\odot}$ of the solar surface. We note, however, that the reduction in $Q$ due to the condition $\Gamma<1$ may itself be mitigated by a further complication, that turbulence within the chromosphere [where $z^{+} \sim z^{-}$ (CvB05)] may lead to the launching of Alfvén waves into the corona with a broad range of perpendicular length scales, extending to very small values (Chandran 2008). Further work is needed to explore how these effects modify the radial profile of the turbulent heating rate. 


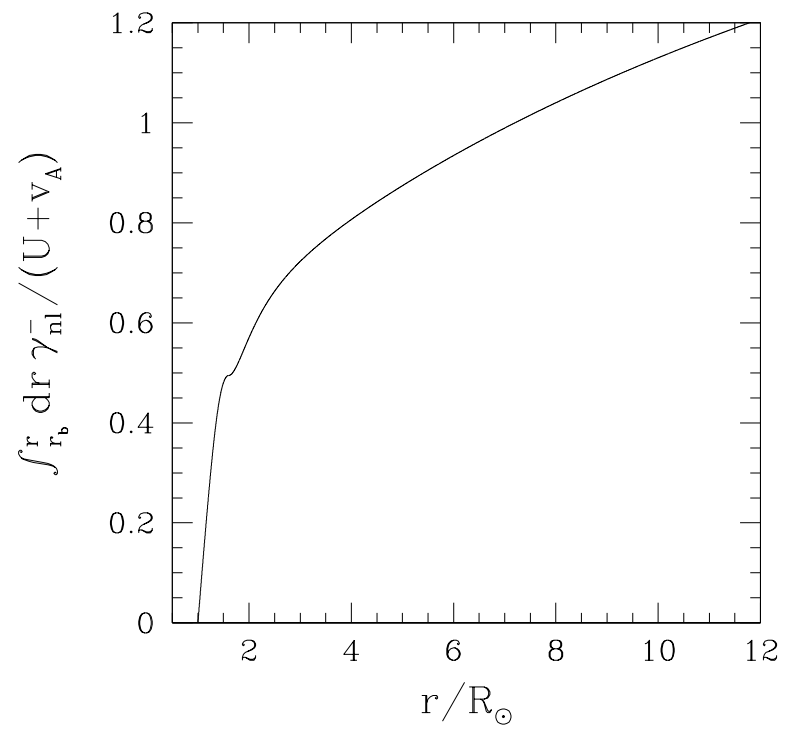

FIG. 9. - The time integral of the shearing rate experienced by a $z^{+}$wave packet between the time it leaves the coronal base at $r=r_{b} \simeq 1 R_{\odot}$ and the time it reaches radius $r$, from equation (43) with $\chi=1$.

In one sense, Figure 9 provides a rough consistency check on models in which the solar wind is heated and accelerated by low-frequency Alfvén-wave turbulence. If $\Gamma$ were $\ll 1$ at $r=r_{a}$, then only a tiny fraction of the Alfvén-wave energy launched from the base of the Sun would dissipate in the region where most of the heating and acceleration of the solar wind takes place. A small fraction of the Alfvén-wave energy would be unable to generate the solar wind, because the Alfvén wave energy flux measured in situ at $r>0.3 \mathrm{AU}$ is much smaller than the total solar-wind energy flux, even allowing for loss of Alfvén-wave energy due to work done on the flow by the ponderomotive force. On the other hand, if $\Gamma$ rose from 0 at $r=r_{b}$ to a value $\gg 1$ at say $r=7 R_{\odot}$, then most of the wave energy would dissipate very close to the Sun (within the radius at which $\Gamma$ reached a value of a few). If the heating rate were to fall too rapidly with $r$, then the heating profile would become inconsistent with UVCS observations that show ion temperatures staying flat or increasing with radius out to at least $3.5 R_{\odot}$ despite adiabatic cooling (Kohl et al 1998; Antonucci, Dodero, \& Giordano 2000).

\section{CONCLUSION}

In this paper, we consider Alfvén-wave reflection and turbulent heating in the solar wind using the Heinemann-Olbert equations, which describe non-compressive MHD fluctuations in an inhomogeneous medium with a background flow parallel to the background magnetic field. We approximate the nonlinear terms in these equations using a simple phenomenological model from D02. Wave reflection plays an essential role in our calculation of the turbulent heating rate, because only outward-propagating waves are generated by the Sun, and turbulent interactions arise only from interactions between oppositely propagating waves (for the lowfrequency, non-compressive Alfvén waves that we consider).

In section 2, we restrict our attention to waves with periods of hours and longer. Our focus on low-frequency waves in this section is motivated by in situ observations showing that most of the wave power is at periods of hours in the space- craft frame. We assume that the energy in inward-propagating waves is much less than the energy in outward-propagating waves, i.e., $z^{-} \ll z^{+}$, an approximation that is appropriate for coronal holes and the fast solar wind at $r<1 \mathrm{AU}$. We also assume that the wave period is longer than the energy cascade time for Sunward-propagating waves, $\sim \lambda / z^{+}$. With the use of these approximations, we solve our model equations analytically to obtain expressions for the rms amplitudes of outward and inward waves as a function of $r$. We also obtain an analytical expression for the radial profile of the turbulent heating rate. There is one free parameter in the heating rate in our low-wave-frequency model: the amplitude of the outward-propagating waves at some fixed reference point. We take this reference point to be the Alfvén critical point $r=r_{A}$, which is at $11.1 R_{\odot}$ in our model solar wind. Interestingly, as in the model of D02, the heating rate does not depend upon the choice of the dominant perpendicular length scale of the turbulence, $\lambda$. Ordinarily, the heating rate is inversely proportional to $\lambda$. However, in our model, the amplitude of the inward-propagating waves is proportional to $\lambda$, because small $\lambda$ leads to rapid turbulent dissipation of the inward waves. As a result, $Q \simeq \rho\left\langle\left(z^{+}\right)^{2}\right\rangle\left\langle\left(z^{-}\right)^{2}\right\rangle^{1 / 2} / 4 \lambda$ becomes independent of $\lambda$, as in equation (5) of D02. Our model can be thought of as generalizing the phenomenological model of D02 by accounting for the solar wind velocity, so that the model can be applied all the way from the coronal base out past the Alfvén critical point.

As discussed in section 2 , one of the approximations in our low-wave-frequency model — that the energy cascade time of Sunward-propagating waves is shorter than the wave period measured in the Sun's frame - breaks down for $r \gtrsim 20 R_{\odot}$ for a wave period of $\sim 3$ hours. In addition, our low-wavefrequency model does not account for waves with periods much shorter than one hour, which may make a significant contribution to the outward wave flux from the Sun (CvB05; but see VV07). To overcome these limitations, we develop an "extended" model in section 3 that approximately accounts for waves with shorter periods and does not require the nonlinear time scale to be shorter than the wave period in the reference frame of the Sun. The main additional ingredient in this extended model is a new free parameter, $\chi$, that models the decrease in the efficiency of wave reflection at shorter wave periods. For appropriate choices of the two free parameters in the model $\left(\chi=0.65\right.$ and $\left.g_{a}=7.2 \times 10^{7} \mathrm{~cm} / \mathrm{s}\right)$, our extended model is successful at matching observations of velocity fluctuations and the Elsässer fields.

Both of the models we have developed provide analytical expressions for the turbulent heating rate that can be used to incorporate Alfvén-wave reflection and turbulent heating into fluid models of the solar wind, both inside and outside the Alfvén critical point. We conclude by summarizing the relative advantages and disadvantages of the two models:

1. The low-wave-frequency model described in section 2 involves a simplistic phenomenological approximation of only the nonlinear terms in the basic equations. The heating rate in the model involves only a single free parameter, $g_{a}$, which determines the amplitude of the outward waves at the Alfvén critical point. On the other hand, one of the assumptions of the model breaks down at $r \gtrsim 20 R_{\odot}$, where the nonlinear time scale becomes longer than the wave period measured in the frame of the Sun (for wave periods of $\sim 3$ hours). In addition, the model does not account for waves with periods much 
shorter than 1 hour, which may contribute significantly to the outward wave flux (CvB05).

2. The extended model described in section 3 does a better job of matching observational constraints on the wave amplitudes, accounts (in a very approximate way) for both long-period waves and waves with periods much less than one hour, and can be applied all the way out to $1 \mathrm{AU}$. On the other hand, this model involves simple phenomenological modeling of both the nonlinear terms and the linear reflection term. The heating rate in the model also involves two free parameters instead of one.

We thank the anonymous referee for a very helpful report. This work was supported in part by NSF Grant ATM-0851005, by DOE Grant DE-FG02-07-ER46372, by NSF-DOE Grant AST-0613622, and by NASA Grants NNX07AP65G and NNX08AH52G.

\section{REFERENCES}

Allen, L. A., Habbal, S. R., \& Hu, Y. Q. 1998, J. Geophys. Res., 103, 6551 Antonucci, E., Dodero, M. A., \& Giordano, S. 2000, Solar Phys., 197, 115 Barnes, A. (1981), Turbulence and Dissipation in the Solar Wind, in Solar Wind Four, edited by H. Rosenbauer, p. 326, Max Planck Institut fr Aeronomie Report No. MPAE-W-100-81-31, Katlenburg-Lindau.

Bavassano, B., Pietropaolo, E., \& Bruno, R. 2000, J. Geophys. Res., 105, 15959

Belcher, J. W., \& Davis, L. 1971, J. Geophys. Res., 76, 3534

Belcher, J. W., Davis, L., \& Smith, E. J. 1969, J. Geophys. Res., 74, 2302

Breech, B., Matthaeus, W. H., Minnie, J., Bieber, J. W., Oughton, S., Smith, C. W., \& Isenberg, P. A. 2008, J. Geophys. Res., 113, A08105

Bruno, R., \& Carbone, V. 2005, Living Rev. Sol. Phys., 2, 4

Chandran, B. D. G. 2008, ApJ, 685, 646

Chandran, B. D. G., Quataert, E., Howes, G. G., Hollweg, J. V., \& Dorland, W. 2009, ApJ, 701, 652

Coleman, P. J. 1968, ApJ, 153, 371639, 1177

Cranmer, S. R., Matthaeus, W. H., Breech, B. A., \& Kasper, J. C. 2009, ApJ, 702,1604

Cranmer, S. R. \& van Ballegooijen, A. A. 2005, ApJS, 156, 265 (CvB05)

Cranmer, S. R., van Ballegooijen, A. A., \& Edgar, R. J. 2007, ApJS, 171, 520

Del Zanna, L., Velli, M., \& Londrillo, P. 2001, A\&A, 367, 705

Dmitruk, P., Milano, L. J., \& Matthaeus, W. H. 2001, ApJ, 548, 482

Dmitruk, P., Matthaeus, W. H., Milano, L. J., Oughton, S., Zank, G. P., \& Mullan, D. J. 2002, ApJ, 575, 571 ("D02")

Dmitruk, P., \& Matthaeus, W. H. 2003, ApJ, 597, 1097

Esser, R., \& Sasselov, D. 1999, ApJ, 521, L145

Feldman, W. C., Habbal, S. R., Hoogeveen, G., \& Wang, Y.-M. 1997, J. Geophys. Res., 102, 26905

Galeev, A. A., \& Oraevskii, V. N. 1963, Sov. Phys.-Dokl., 7, 988

Goldstein, M. L., Roberts, D. A., \& Matthaeus, W. H. 1995, ARAA, 33, 283

Grappin, R., \& Velli, M. 1991, Ann. Geophys., 9, 416
Grappin, R., Velli, M., \& Mangeney, A. 1993, Phys. Rev. Lett., 70, 2190 Heinemann, M., \& Olbert, S. 1980, J. Geophys. Res., 85, 1311

Hollweg, J.V. (1983), Coronal heating by waves, in Solar Wind Five, edited by M. Neugebauer, p. 3, NASA Conf. Publ. 2280, Washington DC.

Hollweg, J. V. 1986, J. Geophys. Res., 91, 4111

Hollweg, J. V., \& Isenberg, P. A. 2002, J. Geophys. Res., 107, 1

Hollweg, J.V., \& Isenberg, P. A. 2007, J. Geophys. Res., 112, A08102

Hollweg, J. V., \& W. Johnson 1988, J. Geophys. Res., 93, 9547

Kadomtsev, B. B. \& Pogutse, O. P. 1974, Sov. JETP, 38, 283

Marsch, E., \& Tu, C.-Y. 1996, Ann. Geophys. 14, 259

Matthaeus, W. H., Zank, G. P., Oughton, S. , Mullan, D. J., \& Dmitruk, P. 1999, ApJ, 523, L93

Roberts, D. A., Goldstein, M. L., Matthaeus, W. H., \& Ghosh, S. 1992, J Geophys. Res., 97, 17115

Schekochihin, A. A., Cowley, S. C., Dorland, W., Hammett, G. W., Howes, G. G., Quataert, E., \& Tatsuno, T. 2009, ApJS, 182, 310

Spruit, H. 1981, "Magnetic flux tubes," in The Sun as a Star, ed. S. Jordan, p. 385, NASA SP-450, Washington DC.

Strauss, H. R. 1976, Phys. Fluids, 19, 134

Tu, C.-Y. 1987, Solar Phys., 109, 149

Tu, C.-Y. 1988, J. Geophys. Res., 93, 7

Tu, C.-Y., \& Marsch, E. 1995, Sp. Sci. Rev., 73, 1

Tu, C.-Y., Pu, Z.-Y, \& Wei, F.-S. 1984, J. Geophys. Res., 89, 9695

Velli, M. 1993, Astron. Astrophys., 270, 304

Velli, M., Grappin, R., \& Mangeney, A. 1989, Phys. Rev. Lett., 63, 1807

Velli, M., Grappin, R., \& Mangeney, A. 1990, Comp. Phys. Comm., 59, 153

Verdini, A., \& Velli, M. 2007, ApJ, 662, 669 (VV07)

Verdini, A., Velli, M., \& Buchlin, E., 2009, ApJL, 700, 39

Viñas, A. F., \& Goldstein, M. L. 1991, J. Plasma Phys., 46, 129

Zank, G. P. \& Matthaeus, W. H. 1992, J. Plasm. Phys., 48, 85 